\title{
Impetus beliefs as default heuristics: Dissociation between explicit and implicit knowledge about motion
}

\author{
MARIA KOZHEVNIKOV and MARY HEGARTY \\ University of California, Santa Barbara, California
}

\begin{abstract}
We examined the extent to which findings from the literature on naive physics and representational momentum studies are consistent with impetus beliefs postulating imparted internal energy as a source of motion. In a literature review, we showed that, for situations in which impetus theory and physical principles make different predictions, representationalmomentum effects are consistent with impetus beliefs. In three new experiments, we examined people's implicit and explicit knowledge of the effect of mass on the rate of ascending motion. The results suggest that implicit knowledge is consistent with impetus theory and is unaffected by explicit knowledge. Expert physicists, whose explicit knowledge is in accord with Newtonian principles, exhibited the same implicit impetus beliefs as novices when asked to respond in a representational momentum paradigm. We propose that, in situations in which an immediate response is required and one does not have specific contextual knowledge about an object's motion, both physics experts and novices apply impetus principles as a default heuristic.
\end{abstract}

Everyday experience provides people with innumerable opportunities for interacting with moving objects. For example, in order to catch a ball, we have to anticipate its trajectory and place our hands in the right position in space and time to interrupt its motion. How accurate are our predictions about objects' trajectories? What sources of information do people use to predict objects' motions?

The above questions have received widespread attention in experimental psychology and physics education studies. These studies vary widely in their methods and experimental techniques. On one end of a continuum are so-called naive physics studies related to the fields of cognitive psychology and physics education (e.g., Clement, 1982, 1983; McCloskey, 1983; McCloskey, Caramazza, \& Green, 1980; McCloskey \& Kohl, 1983; McCloskey, Washburn, \& Felch, 1983). Most of these studies examine people's beliefs about motion that are verbalizable and consciously accessible and may be considered to be explicit. The general conclusion from naive physics studies is that many people hold erroneous beliefs concerning fundamental laws of motion that are rather similar to medieval impetus beliefs (McCloskey, 1983). In particular, people hold a mistaken notion that a force applied to an object gives it a store of energy (impetus) that serves to maintain the motion after the object has been released.

\footnotetext{
This research was supported in part by the Off ice of Naval Research under Contract N00014-96-10525 to the second author. We thank Michael Grace-Martin for programming the displays in Experiments 1 and 3. Correspondence concerning this article should be sent to M. Kozhevnikov, Department of Psychology, Rutgers University, Smith Hall, 101 Warren Street, Newark, NJ 07102 (e-mail: maria@psychology.rutgers.edu) or M. Hegarty, Department of Psychology, University of California, Santa Barbara, CA 93106 (e-mail: hegarty@psych. ucsb.edu).
}

On the other end of the continuum are a number of cognitive psychology studies that examine observers' responses on a memory task, which is accomplished by nonconscious processes and based on implicit perceptual knowledge (e.g., Finke, Freyd, \& Shyi, 1986; Freyd, 1987; Freyd \& Finke, 1984). The major finding of these studies is that if an observer views an object undergoing implied or apparent motion and the object suddenly disappears, memory for the object's final position is shifted forward in the direction of motion. The general conclusion from these studies is that the perceptual system embodies a principle analogous to physical momentum (i.e., continuing movement of an object until acted upon by a force). This phenomenon has been called representational momentum (RM). Freyd $(1987,1992)$ has suggested that RM results from an adaptive internalization of physical momentum into our cognitive-perceptual system. Hubbard $(1995 b, 1998)$ has proposed that RM reflects internalization of environmentally invariant physical principles (such as gravity, friction, and centripetal force).

To what extent might implicit perceptual knowledge about motion differ from explicit conceptual knowledge? Several studies have suggested that from daily interactions with moving objects, people develop perceptually based knowledge about motion that is much more accurate than their naive verbal-cognitive concepts of motion and that this implicit knowledge follows a different developmental course (e.g., Krist, Fieberg, \& Wilkening, 1993). Similarly, Freyd (1987) originally suggested that our implicit intuitive knowledge about motion, as measured in RM studies, reflects internalization of physical principles. This implicit knowledge may be cognitively impenetrable and much more accurate than our explicit conscious knowledge (see also Hubbard, 1998). 
There are several problems, however, with the conclusion that implicit knowledge of motion is more accurate than explicit knowledge. First, the origin of the strongly held impetus beliefs found in naive physics research remains unclear. If, over the course of evolution, we have acquired implicit knowledge of physical principles and, during formal physics instruction, we are taught those principles explicitly, why do impetus ideas persist even in college physics students? Second, implicit knowledge about motion does not always correspond to the actual behavior of physical systems, and a number of additional findings suggest that the momentum analogy may be misleading (e.g., Cooper \& Munger, 1993; Freyd \& Jones, 1994; Verfaillie \& D'Y dewalle, 1991). For instance, Freyd and Jones studied explicit and implicit judgments of the trajectory that a ball would take when exiting a spiral tube. The majority of participants selected the correct trajectory when asked explicitly. However, participants' implicit knowledge, expressed in an RM paradigm, was incorrect and more consistent with impetus predictions. That is, participants exhibited greatest RM not along the correct straight path, but along the incorrect spiral path. Freyd and Jones suggested that, at the perceptual level, participants might attribute an internal force (impetus) to the moving ball that causes the ball to follow a spiral path even after leaving the tube.

In the present study, we propose that implicit knowledge about motion is not in accord with physical principles but, rather, reflects impetus notions derived from our everyday experience. We suggest that this implicitimpetus knowledge operates despite conscious beliefs to the contrary and often causes people to be inaccurate in naive physics tasks. The environment is filled with frictional forces, and most moving objects seem to stop of their own accord. Reliance on the implicit impetus notion that moving objects somehow lose their internal energy allows us to respond quickly and accurately in a variety of everyday situations. Nevertheless, the reliance on impetus principles may lead to systematic errors in situations that do not occur very often in everyday life (e.g., balls emerging from a spiral tube). We propose that under conditions in which implicit impetus knowledge leads us astray, people with correct explicit knowledge of physics laws can produce the correct answer if they have enough time to reflect. However, in situations in which an immediate response is required, both physics experts and novices apply impetus principles as a default heuristic.

To provide evidence for the above hypothesis, we first conducted a thorough review of the literature on naive physics and RM. This review revealed that for situations in which impetus theories and Newtonian principles make different predictions, RM effects are consistent with impetus beliefs. We then compared implicit knowledge about motion in a gravitational field (expressed in an RM paradigm) with verbal conceptual knowledge (expressed in explicit judgments). Specifically, we assessed people's predictions of how fast two different masses will ascend in a gravitational field when launched vertically with the same initial velocity, a situation rarely experienced in everyday life. Furthermore, we examined whether expert physicists, whose explicit conceptual knowledge is in accord with Newtonian principles, exhibit the same implicit impetus knowledge as novices when asked to respond quickly in the above situation. If people intuitively apply impetus principles as a default heuristic, we expect that there will be no significant differences between the RM effects exhibited by expert and novice physicists and that these effects will be in accord with impetus, but not Newtonian, principles.

\section{LITERATURE REVIEW}

In this section, we will review the historical development of theories of motion, naive physics studies, and RM research. The goal of this review is to examine the kinds of knowledge about motion that are incorporated into our perceptual and cognitive systems.

\section{Historical Development of Theories of Motion}

We begin with a historical review of theories of motion. This reveals the range of explicit theories of motion that have been articulated in the history of science and also provides a benchmark against which to compare the conceptions of motion held by different individuals.

Theories about motion predate the ancient Greeks, but Aristotelian and medieval impetus theories influenced physical science until the time of Newton. According to Aristotle's theory, all observed motion of inanimate objects falls into two categories: natural motion and violent motion. Natural motion occurs in the absence of forces, because bodies seek to reach their "natural place," in which they will be in a state of rest. The natural motion of the heavy elements (earth and water) is to fall downward. The natural motion of the light elements (air and fire) is to rise. According to Aristotle, violent motions are those produced by forces. An object remains in violent motion only so long as it is in direct contact with an external mover. To account for the movements of projectiles that are not in direct contact with any "observable" mover, Aristotle suggested that the medium itself (air or water current moving rapidly backward) pushes the object forward (see Dijksterhuis, 1961).

Finding an Aristotelian explanation unsatisfactory for projectile motion, several medieval philosophers proposed the concept of impetus. The first to discuss impetus was John Philoponus, a 6th-century Greek scholar, and the concept was developed further in the 11th century by Avicenna and in the 14th century by the philosophers Franciscus di Marchia, Jean Buridan, and Nicole Oresme (see Dijksterhuis, 1961; Franklin, 1978). In contrast to Aristotelian physics, in which the force responsible for motion was proposed to be external to the moving object, impetus theory assumed that the motion is maintained by a force internal to the object, which is acquired when the object is set in motion. There are some 
differences between different versions of impetus theory, but all of them postulate that the act of setting an object in motion imparts to the object an impetus (i.e., an internal force) that maintains the motion after the object has been released. Furthermore, most versions of impetus theories posit the following: (1) A moving object's impetus progressively dissipates and, as a result, the object decelerates and comes to a stop. The impetus is counteracted by the resistance of the medium in which the motion takes place and, in the case of a body thrown upward, also by gravity, which tends to convey it downward. (2) The nature of the motion is determined by impetus, so that in one case it can maintain upward motion of projectiles, in another sideways motion, and in a third case motion in a circle. Medieval thinkers even translated the concept of impetus into a more precise form of proportionality to mass and velocity, the same mathematical representation as the concept of momentum in Newtonian physics. However, impetus was regarded as the cause of motion, whereas in Newtonian mechanics, momentum is usually seen only as a symptom of motion-that is, a quantity characterizing it. Furthermore, a qualitative distinction between a state of rest (absence of impetus) and a state of motion (presence of impetus) is contradictory to the principles of Newtonian mechanics.

Hundreds of years after impetus theories were proposed, Galileo and Newton demonstrated that objects remain in motion at a constant velocity or at rest unless acted upon by an external force. No force is required to keep an object in motion at a constant velocity. Any object that is not accelerating can be equally described as at rest or as in constant velocity motion, depending on the choice of a frame of reference.

\section{Naive Physics Studies}

To what extent are Aristotelian and impetus principles reflected in explicit ideas about projectile motion? Examination of naive observers' explicit responses to questions concerning projectile motion reveals that these responses are rather similar to medieval impetus notions (e.g., Clement, 1982, 1983; McCloskey, 1983; McCloskey et al., 1980; McCloskey \& Kohl, 1983; McCloskey et al., 1983).

The belief system of young children includes Aristotelian notions of natural motion. For instance, some younger children (ages 7-10) believe that falling has an initial cause-namely, a loss of support-but is a natural motion, since there is no need for any force or agency for it to continue (Eckstein \& Kozhevnikov, 1997; Eckstein \& Shemesh, 1993; Ogborn, 1985). However, most students, beginning about Grades 5-6 (age, 11-12), acquire beliefs resembling impetus rather than Aristotelian theories (Eckstein \& Kozhevnikov, 1997) and continue to hold these beliefs even after physics instruction. Although some researchers have characterized older children's conceptions as Aristotelian, this term was used inappropriately, and the belief systems of most students are closer to medieval impetus theory (see Halloun \& Hestenes, 1985, for a detailed discussion).
Although researchers have often emphasized the "lack of a well-specified naive (impetus) physics theory" (Ranney, 1994, p. 495), it is incorrect to characterize impetus beliefs as a chaotic set of ideas. There are many different versions of impetus theory, but all of them postulate the same fundamental idea that the act of setting an object in motion imparts to the object an internal force (impetus). ${ }^{1}$ Therefore, we consider naive beliefs to reflect impetus principles if they are based on the assumption that motion is maintained by a force internal to an object. On the basis of a review of naive physics research, the following impetus views common to people's naive ideas and medieval impetus theory can be identified.

First, a large proportion of people believe that an object must be directly pushed or pulled to acquire impetus. That is, an object that is merely carried in a horizontal direction by another moving object does not acquire impetus and, therefore, will fall straight down after being released (e.g., Eckstein \& Kozhevnikov, 1997; Fishbein, Stavy, \& Ma-Naim, 1989; McCloskey et al., 1983). For instance, Fishbein et al. found that $50 \%$ of participants believed that a ball carried by an airplane would fall straight down if released, but only $13.6 \%$ believed that a ball launched over a precipice would fall straight down.

Second, many people believe that impetus is selfexpending and that, even in the absence of any external influences, an object's impetus progressively dissipates, so that the object decelerates and comes to a stop. Others believe that impetus is exhausted only by frictional forces. Both of these theories can be seen in history. Buridan, for instance, argued that impetus is not selfexpending, whereas Franciscus di Marchia and Oresme stated that impetus dissipates even in the absence of friction and air resistance (see McCloskey, 1983, for a review).

Third, many people believe that the nature of motion is determined by impetus - for example, that a "force of throw" acts on an object thrown vertically upward (Clement, 1983; diSessa, 1983, 1988, 1993; Viennot, 1979). These people believe that this force must be greater than gravity; otherwise, the object would immediately fall down. This impetus view is similar to one held by Galileo in the early stages of his research (see Clement, 1983). As was described above, many people also believe that an object constrained to move in a curved path acquires a curvilinear impetus that causes the object to follow a curved trajectory for some time after the constraints on its motion are removed (McCloskey, 1983; McCloskey et al., 1980; McCloskey \& Kohl, 1983). Many impetus theorists also postulated a circular impetus that served to maintain the motion of a wheel or sustain the rotation of the celestial objects (see Franklin, 1978).

Fourth, some people believe that there is interplay between impetus and gravity-that is, gravity does not affect an object until its original impetus falls below some critical level. Thus, for instance, a projectile fired horizontally will follow a straight horizontal trajectory for a while, but its impetus continually dissipates, and at some moment, the projectile, while still moving forward, will 
begin to fall (McCloskey, 1983; Ranney, 1994). Some medieval theorists also believed that gravity would not affect an object until its impetus had been entirely or partially expended, whereas others held that gravity would affect an object's motion regardless of how much impetus it had (see a review in Dijksterhuis, 1961).

The parallel between students' naive conceptions and historical views about motion is striking and casts doubt on the idea that impetus beliefs are just the result of a fragmented understanding of the world of physics. It seems that "to this day every student of elementary physics has to struggle with the same errors ... and on a reduced scale... history repeats itself every year" (Dijksterhuis, 1961, p. 30).

Consistency of impetus beliefs. There is still a debate in the literature as to whether impetus notions present a consistent belief system (e.g., McCloskey, 1983) or whether they are just the result of a fragmented and situational understanding of physical phenomena (e.g., diSessa, 1983, 1988; Ranney, 1988, 1994). For instance, Ranney and Thagard (1988) found that the same participant might give responses reflecting different motion models (impetus or Newtonian) to different problems, with no correlation of response types across problems (see also Cooke \& Breedin, 1994, and Ranney's, 1994, commentary). That is, people may hold impetus beliefs for McCloskey's curved tube problem, while at the same time giving Newtonian responses regarding horizontal projectile motion. As a result, diSessa (1988) has proposed that instead of a consistent set of beliefs, people might have "a fragmented collection of ideas, loosely connected and reinforcing, having none of the commitment or systematicity that one attributed to theories" (p. 50). Cooke and Breedin suggested that trajectory judgments and explanations are constructed on the fly, with the use of a variety of problem features and knowledge fragments.

Although there is abundant evidence that people's ideas about motion are not completely coherent, this does not imply that there is no theoretical framework that can make accurate predictions about people's judgments of objects' trajectories. Although there is little doubt that contextual cues are critical in trajectory judgments (Catrambone, Jones, Jonides, \& Seifert, 1995; Cooke \& Breedin, 1994; Kaiser, Jonides, \& Alexander, 1986), the possibility that people's responses might be additionally based on fairly systematic impetus beliefs cannot be ruled out. For example, people may draw on specific experiences to make judgments about object motion in some situations and may fall back on systematic impetus notions in other kinds of situations.

Impetus as the basis for reasoning in unfamiliar situations. People are able to reason appropriately about motion problems related to specific concrete experiences. For example, Kaiser et al. (1986) found that participants produced significantly more correct predictions for the trajectory of water emerging from a curved hose than for a ball emerging from a curved tube. Further- more, the way in which a problem is presented (in a static diagram or a dynamic animation) or the complexity of the situation may significantly affect task performance (Kaiser, Proffitt, \& Anderson, 1985; Kaiser, Proffitt, Whelan, \& Hecht, 1992). However, although people make correct predictions in familiar situations, such as water emerging from a curved hose, they are unable to generalize this knowledge to subsequent unfamiliar problems, such as a ball emerging from a curved tube (Catrambone et al., 1995; Kaiser et al., 1986). Catrambone et al. concluded that a previous instance must be very superficially similar to a familiar problem to be used as an analogy. Otherwise, people fall back on their impetus beliefs as the basis of their reasoning.

In summary, naive physics research has shown that explicit theories of motion often reflect the basic impetus beliefs. Although contextual cues affect people's judgments of motion, the possibility that people's responses might be additionally based on impetus beliefs cannot be ruled out. For example, when a problem does not elicit a familiar experience, people might systematically fall back on naive impetus responses. In all of the cases in which impetus beliefs are shown, people do not have situationspecific experiences that may help them with a problem solution. They do not often watch balls emerging from curved tubes, bombs thrown from airplanes, or launching projectiles. In this paper, we propose that rather than being unsystematic or "theories of motion on the fly," impetus beliefs reflect a systematic set of beliefs, consistent across individuals, that people fall back on when they do not have specific contextual experience.

\section{Representational Momentum Studies}

We now consider the extent to which impetus theories are incorporated into implicit knowledge about motion, as revealed by RM studies.

When an observer views an object undergoing implied or apparent motion and the object suddenly disappears, memory for the object's final position is shifted forward in the direction of motion (e.g., Finke \& Freyd, 1985; Finke et al., 1986; Freyd, 1987; Freyd \& Finke, 1984). Freyd (1987) originally concluded that this memory distortion results from dynamic visual representations that reflect an internalization of the inertial properties of real-world object motion. Consistent with this prediction, Freyd and Finke found that the magnitude of RM increased linearly with the velocity implied by the static displays. Freyd \& Johnson (1987) also showed that for short retention intervals, the rate of increase in RM was proportional to the implied velocity of the inducing display, as is predicted from the analogy to physical momentum. On the basis of these results, Freyd (1987, 1993) suggested that RM reflects a spatiotemporal coherence between the external world and internal mental representations.

In addition, Hubbard (1990; Hubbard \& Bharucha, 1988) found that memory for descending objects exhibits greater forward displacement than does memory 
for ascending objects and that memory distortion for horizontally moving objects is also displaced downward below the path of motion. This is consistent with realworld phenomena (i.e., descending objects accelerate, ascending objects decelerate, and horizontally projected objects fall in a parabola). Furthermore, when a horizontally moving target slides along the upper edge of a larger stationary surface or crashes through a barrier, the RM decreases, which is consistent with the operation of frictional forces in a real world (Hubbard, 1995a). Hubbard (1995a, 1995b, 1998) has proposed that the above memory distortions might reflect internalization of environmentally invariant physical principles (such as gravity and friction).

Although a number of additional studies (e.g., Cooper \& Munger, 1993; Freyd \& Jones, 1994; Verfaillie \& D'Ydewalle, 1991) questioned the momentum analogy, the results described above seem to suggest that memory distortions, at least in some situations, follow the principles of Newtonian physics. However, in all these situations, impetus theory makes the same predictions as Newtonian physics. First, impetus cannot be instantly dissipated, and objects cannot be immediately stopped unless they encounter an opposite force. Second, the mathematical representations of impetus and physical momentum are similar, so the effects of velocity on memory distortions cannot differentiate whether impetus or real physics principles are internalized into our representational system. Third, according to impetus theory, an object's impetus progressively dissipates as a result of its counteraction with gravity, in the case of ascending motion, and consequently, the object decelerates. In the case of descending motion, an impetus acts in the same direction as gravity, and the object accelerates.

Therefore, on the basis of the experiments described above, it is impossible to conclude unambiguously that, in these situations, memory distortions follow correct physics principles. If impetus principles were internalized, they would produce exactly the same memory distortions. To discriminate whether implicit knowledge is based on impetus or Newtonian principles, we need to examine cases in which impetus and physical principles give different predictions. We now turn to a consideration of these cases.

First, a ball shot out from a spiral tube will follow a straight trajectory according to Newtonian principles and a spiral path according to impetus predictions. If people have accurate implicit knowledge of the underlying physical principles, the RM for the ball should be produced along the correct straight-line path, and not along the spiral path. However, as was previously noted, Freyd and Jones (1994) found greater RM along the spiral path. In an attempt to reconcile these results with the hypothesis that physical principles are internalized in our representational system, Hubbard (1996, p. 1050) introduced the concept of representational centripetal force. That is, the mental representation of an object follows a curved trajectory after exiting the tube because of an inability to instantly stop the action of centripetal force on an object, much as RM results from the inability to instantly stop the represented motion of the object. The concept of representational centripetal force introduced by Hubbard is close to the idea of curvilinear impetus, which according to impetus theory, cannot be instantly dissipated and causes the ball to move in a curved path after exiting the tube.

Second, if RM reflects correct laws of physics, and not impetus principles, the magnitude of distortion in memory for horizontally moving projectiles shot out from the same height should be the same in the downward direction, regardless of their horizontal velocity. Hubbard (1995a) examined whether judgments of the locations of horizontally moving targets were influenced by implied friction between the targets and the surfaces. Targets sliding along the upper edge of a single stationary surface exhibited less forward displacement than did targets not moving along a surface. After the targets slid along the upper edge of a single stationary surface, horizontal RM was decreased, and downward memory distortions increased, in comparison with those targets that were not in contact with a surface. Hubbard (1995a) suggested that observers' representations reflect an "interaction between friction and gravity." According to Hubbard, the representational system exploits the pattern that, as an object descends along a parabola, the vertical component of motion increases as the horizontal component decreases, making us believe that the vertical and the horizontal components are interrelated. This pattern is remarkably similar to medieval impetus ideas that the more impetus is exhausted by friction, the more the force of gravity pulls the object down. According to correct physics laws, effects of gravity (producing downward displacement) are independent of horizontal velocity (in this case, produced by different amounts of friction).

Third, if correct physics laws are incorporated into the perceptual system, the memory displacement for objects of different mass falling in a gravitational field should be the same. Hubbard (1997) examined how target size influences the magnitude of memory displacement for falling objects. It was assumed that larger targets would be perceived as more massive, based on a long tradition of research on the size-weight illusion (e.g., Jones, 1986; Koseleff, 1957; Masin \& Crestoni, 1988). Hubbard (1997) found an effect of target size on memory displacement along the vertical axis, aligned with implied gravitational attraction. With descending motion, larger targets exhibited larger forward memory displacement than did smaller targets, whereas with ascending motion, larger targets exhibited smaller forward memory displacement. This is inconsistent with the behavior of a physical system, either in an ideal frictionless or in a real physical world, but reflects impetus ideas (see the detailed explanation of impetus and physical principles predictions for these cases in Table 1). To interpret the above results, Hubbard (1997) used the concept of representational gravity, suggesting that our representational system appears to reflect the phenomenological 
consequences of the physical principles of gravitational attraction. The concept of representational gravity is not consistent with the actual consequences of the gravitational attraction, but it strikingly resembles impetus ideas.

In summary, in situations in which impetus principles and Newtonian principles make different predictions, memory displacements are consistent with impetus principles. This suggests that the representational system does not incorporate or extrapolate the effects of environmentally invariant physical principles per se. Rather, as Hubbard (1998) recently suggested, the representational system appears to reflect the subjective or phenomenological consequence of those physical principles. Moreover, we argue that these subjective consequences, although very different, are all based on the impetus idea that energy is imparted to objects and cannot dissipate instantly. Although all situations that discriminate between impetus and Newtonian principles have obviously not been studied, what is important is that we did not find any case in the literature that reported a situation in which memory distortions reflected Newtonian theory, and not impetus theory.

\section{Dissociation Between Implicit and Explicit Knowledge}

Our review of the literature on naive physics and RM suggests that mental representations are composed of (1) conscious and explicit knowledge regarding objects' motion that can be either correct or incorrect, depending on knowledge of physics, and (2) nonconscious and implicit knowledge, which is influenced by our perceptual experience and reflects impetus notions. We now turn to the question of whether implicit knowledge is consistent or inconsistent with explicit knowledge.

There is a debate in the literature about whether the RM effect is cognitively penetrable or perceptually modular (Finke \& Freyd, 1989; Hubbard \& Bharucha, 1988). On the one hand, specific context and characteristics of the stimulus objects can affect RM. For example, Reed and Vinson (1996) demonstrated that RM was greater for ascending motion when the target was a drawing of a rocket than when the target was a drawing of a church steeple. On the other hand, there is evidence that RM is resistant to viewers' conscious attempts to control it (Freyd, 1987) and thus cannot result from conceptual knowledge alone. Furthermore, Freyd and Jones (1994) found that $\mathrm{RM}$ for a ball emerging from a curved tube follows a curved trajectory, despite explicit knowledge that it should follow a straight trajectory.

To clarify the relation of explicit knowledge to implicit knowledge, it is important to compare the implicit beliefs of expert and novice physicists. If implicit knowledge is cognitively impenetrable by explicit knowledge of physics, RM should be consistent with impetus ideas for expert physicists, as well as for novices.

\section{EMPIRICAL STUDY}

We examined people's explicit and implicit knowledge of the effect of mass on the rate of ascending mo- tion in three experiments. Ascending motion is a case in which Newtonian physics and impetus beliefs clearly make different predictions; it is not often experienced in everyday life, and it has not been studied extensively in the naive physics or RM literature.

Table 1 shows the predictions of Newtonian physics and impetus beliefs for the effects of object mass on ascending and descending motion. It is important to consider these effects in both the real world, in which air resistance plays a role, and in a more "ideal" situation, often considered in physics instruction (e.g., in a vacuum), in which the effects of air resistance can be ignored.

According to Newtonian physics, in the absence of air resistance, all objects move (fall or rise) at the same rate, regardless of mass. In the presence of air resistance, a more massive object will either fall or rise faster than a less massive object of the same shape, because more massive objects are influenced less by the force of air resistance. The mass of an object is proportional to its density and volume - that is, one object can be more massive than another object owing to its higher density (when the size is the same) or to its larger size (when the density is the same). Even in the case of two objects with different sizes and the same density, the more massive (larger) object will move at a higher rate, because the effect of increased mass (proportional to the cube of $L$, where $L$ is the length of an object side) will always exceed the effect of increased air resistance (proportional to the square of $L$ ). ${ }^{2}$

In the case of descending motion in the presence of air resistance, impetus beliefs and Newtonian mechanics give the same predictions, although on a different basis. According to impetus theorists, gravity not only causes a descending object to fall, but also imparts to it an impetus, which acts in combination with gravity. The object is moved by constant gravity and continually growing impetus, so that it moves faster and faster. Heavier objects acquire more impetus as a result of gravity and thus accelerate faster (see Dijksterhuis, 1961, for a historical review of impetus theories).

In the case of ascending motion, however, predictions of Newtonian mechanics and impetus theory are opposite. According to impetus theory, a more massive object ascends more slowly. At every moment of time, gravity interacts with continually decreasing impetus. A more massive object loses its impetus faster and therefore decelerates faster and ascends more slowly than a lighter object. According to Newtonian mechanics, a more massive object decelerates at the same rate (in the absence of air resistance) or at a slower rate (in the presence of air resistance) than a lighter object.

We conducted three experiments. The goal of Experiment 1 was to investigate people's implicit knowledge about ascending motion by examining whether they exhibit less RM for larger targets than for smaller ones when the targets ascend in a gravitational field. The goal of Experiment 2 was to examine explicit conceptual knowledge of people without physics training about objects' motion in a gravitational field. The goal of Experiment 3 was to examine how explicit abstract knowledge influences im- 
Table 1

Newtonian Physics and Impetus Predictions for

the Case of Descending and Ascending Motion

\begin{tabular}{|c|c|c|}
\hline Principles & Descending Motion & Ascending Motion \\
\hline $\begin{array}{l}\text { Newtonian physics } \\
\text { (in the absence of } \\
\text { air resistance) }\end{array}$ & $\begin{array}{l}\text { All physical objects, regardless } \\
\text { of mass and shape, fall with the } \\
\text { same acceleration. }\end{array}$ & $\begin{array}{l}\text { All physical objects, regardless of } \\
\text { mass and shape, rise with the same } \\
\text { acceleration. }\end{array}$ \\
\hline $\begin{array}{l}\text { Newtonian physics } \\
\text { (in the presence of } \\
\text { air resistance) }\end{array}$ & $\begin{array}{l}\text { More massive objects are } \\
\text { influenced less by the force of } \\
\text { air resistance and, as } \\
\text { a consequence, descend faster than } \\
\text { less massive objects of the same } \\
\text { shape. }\end{array}$ & $\begin{array}{l}\text { More massive objects are } \\
\text { influenced less by the force of air } \\
\text { resistance and, as a consequence, } \\
\text { ascend faster than less massive } \\
\text { objects of the same shape. }\end{array}$ \\
\hline $\begin{array}{l}\text { Impetus theory } \\
\text { (regardless of air } \\
\text { resistance) }\end{array}$ & $\begin{array}{l}\text { More massive objects accelerate } \\
\text { faster. Gravity imparts an } \\
\text { impetus }(m v) \text { to a descending } \\
\text { object, which then moves it in } \\
\text { combination with gravity }(m g) \text {. } \\
\text { The more massive the object, the } \\
\text { faster it falls. }\end{array}$ & $\begin{array}{l}\text { More massive objects accelerate at } \\
\text { a slower rate. An object's initial } \\
\text { impetus continually dissipates } \\
\text { because it is overcome by the effect } \\
\text { of gravity. The more massive the } \\
\text { ascending object, the more gravity } \\
\text { counteracts its impetus. }\end{array}$ \\
\hline
\end{tabular}

plicit knowledge by comparing RM for ascending targets of different sizes between people with different levels of expertise in physics.

In all three experiments, we focused on the case of ascending motion, for the following reasons. First, as is shown in Table 1, impetus theory and Newtonian mechanics make the same predictions regarding descending motion, so only the case of ascending motion can discriminate which of these theories best describes implicit knowledge of mechanics. Second, in everyday life, a situation in which two different masses begin to rise with the same initial velocities is much more rarely experienced than a situation in which two different masses begin to fall with the same initial velocity equal to zero. This allows us to examine the effect of abstract conceptual knowledge (and not that resulting from perceptual experience) on RM displacement. Third, in contrast to descending motion, very few studies (e.g., Clement, 1983; Viennot, 1979) have examined beliefs about ascending objects. Clement (1983) found that a majority of his participants exhibited impetus views in this situation; they believed that a "force of throw" acts on an object thrown vertically up. We examined these beliefs further with regard to the ascension rates of objects of different mass.

Finally, Freyd and Jones' (1994) results regarding RM for the spiral tube problem can be explained by the fact that the momentum occurs along a path that is consistent with the inducing display (Freyd \& Jones, 1994; Hubbard $\&$ Bharucha, 1988). That is, simple pattern completion or good continuation would place the momentum along the spiral path. In order to deal with this possible alternative explanation, it is important to examine a situation in which RM effects cannot be explained by path completion. Ascending motion is such a situation, because it predicts different velocities along the same path, rather than different trajectories.

\section{Experiment 1}

In Experiment 1, we studied RM for ascending targets of different sizes. As was described above, Hubbard
(1997) studied the effects of target size on RM while a target moves in the vertical direction and found that larger ascending targets exhibited less forward displacement, as compared with smaller ascending targets. In Hubbard's (1997) experiments, the velocity of a target stimulus was constant within each trial. But in the real world, objects do not move upward with constant velocity, as long as no internal-driven forces (such as the force of a rocket engine) act upon the object. Therefore, motion at constant velocity in a vertical direction might not be perceived as motion of a free object in a gravitational field. In our experiments, we examined RM in a situation in which we modeled the deceleration of objects in a real gravitational field. We hypothesized that even in this more realistic simulation, there would be more RM for smaller ascending objects than for larger ascending objects, reflecting impetus principles.

\section{Method}

Participants. The participants were 9 undergraduate psychology students recruited from the psychology subject pool at the University of California, Santa Barbara. One of these students had taken physics courses at the college level, 4 students had taken general physics courses at high school, and the other 4 students had not received any formal physics instruction.

Stimuli and Apparatus. The stimuli were programmed in Macromedia Director and were displayed by a PowerMacintosh $8500 / 180$ computer on a $10 \times 13$ in. monitor with a viewing distance of approximately $18 \mathrm{in}$. The participants' responses and reaction times were recorded by the computer. The target stimuli were filled black squares with side lengths of 0.4 and 0.8 in., respectively, presented on a white background. In each trial, a square moving vertically upward was displayed on the computer screen by a sequence of six successive frames with a $150-\mathrm{msec}$ stimulus duration and a $150-\mathrm{msec}$ interstimulus interval. The velocity of the square was changed from frame to frame according to the following formula:

$$
d v / d t=-g-k \times v
$$

corresponding to the following shifts along the axis of motion $(y$-axis):

$$
\begin{aligned}
y(t)= & (-1 / k) \times\left(v_{0}+g / k\right) \\
& \times[(\exp (-k \times t)-1)]-(g / k) \times t,
\end{aligned}
$$


where $v$ is the velocity, $g$ is the gravitational constant, $k$ is the velocity damping coefficient (e.g., air resistance coefficient divided by mass) and $v_{0}$ is the initial velocity (see Bronson, 1994). The initial velocity $v_{0}$ of a target was 634 pixels per second, the friction coefficient $k$ was $0.2, \Delta t$ was $150 \mathrm{msec}$, and the gravitational acceleration $g$ was 275 pixels $/ \mathrm{sec}^{2}$. The values of the above constants were chosen to make the motion of a target look as natural as possible on the computer screen and were not equal to real-world constants. (For instance, with actual gravitational acceleration $g=9.8 \mathrm{~m} / \mathrm{sec}^{2}$, a target would move too fast to be shown on the computer screen as a sequence of successive presentations.) The target deceleration was displayed by varying the distance between successive presentations of the target by $91,83,74,65$, and 56 pixels, in that order.

The ascending target crossed approximately 5 in. (74 pixels per inch) before it vanished. The final frame in the sequence was followed by a test frame (seventh frame) with a retention interval of $150 \mathrm{msec}$ (the same as the interstimulus interval). The square depicted in the test frame was shifted slightly forward in the direction of motion, was shifted slightly backward in the direction of motion (the shift was 5 or 10 pixels), or was in the same position as the final square.

Procedure. The participants were first given 12 practice trials at the beginning of the session, drawn randomly from the experimental trials. They were given the following instructions:

In each trial, a vertically moving square will be displayed on the computer screen by a sequence of successive frames. A square in a trial may be large or small. Your task is to attend to all stimulus frames in a sequence and remember the square's final position in a sequence. The final frame in the sequence will be followed by a test frame. The square depicted in the test frame is either in the same position as the final square, is shifted slightly backward in the direction of motion, or shifted slightly forward in the direction of motion. You should remember the square's final position, compare it to the test square's position and indicate whether the two positions are the same. If the two frames are at the identical position, press the " $i$ " key on the keyboard. If the test square is shifted backward or forward in the direction of motion, press the "d" key on the keyboard, which means that the two positions are different. The test square will remain on the screen until you respond. Although you should respond as quickly as possible, first of all, try to be as accurate as possible.

First, the participant received 20 blocks of 12 trials (240 trials total). These trials consisted of equal numbers of same trials, trials in which the test square was shifted forward 5 pixels (which constitutes $9 \%$ of the final shift between the fifth and the sixth frames), and trials in which the test square was shifted backward on 5 pixels. Then, the participant received an additional 20 blocks of trials (240 trials, overall) consisting of same trials and forward and backward trials in which the test square position was shifted by 10 pixels (18\% of the final shift between the fifth and the sixth frames). ${ }^{3}$ The participant initiated each block of trials by clicking on a "continue" sign that appeared on the screen at the end of the previous block.

\section{Results and Discussion}

We predicted that more RM would be observed for small targets than for large targets, reflecting the incorporation of impetus principles into the perceptual system.

To compare the effect of object mass (size) on RM, we used Reed and Vinson's (1996) method of calculating $\mathrm{RM}$. According to this method, RM is calculated by subtracting each participant's proportion of same responses for the 5-pixel shift of the test frame that is inconsistent with implied motion (downward 5-pixel shift) from the proportion of same responses for the 5-pixel shift that is consistent with the implied motion (upward 5-pixel shift). The RM effect was calculated separately for small and large targets. The mean RM effect for small ascend- ing targets was $0.35(S D=0.19)$, indicating that the participants were more likely to answer same to the upward 5-pixel shift than to the downward 5-pixel shift. There was no significant RM effect for large ascending targets (the mean RM effect for the large target was $0.01, S D=$ $0.20)$. As was predicted, a paired-sample $t$ test revealed a significant effect of target size: $[t(8)=2.48, p<.03$ ] The small ascending target produced significantly more RM than did the large ascending target.

Reed and Vinson's (1996) method of computing RM does not use all the data, but only the data for probes of \pm 5 pixels. A more conservative and commonly used measure of RM is to calculate the shift in the peak of the quadratic regression across all probes (cf. Freyd, 1987). To show the reliability of the effect of mass on RM, the data were also subjected to a quadratic regression. Solving for the peak of the curve revealed positive RM for the small target (a shift of 2.9 pixels) and insignificant $\mathrm{RM}$ for the larger target (0.6 pixels), as is displayed in Figure 1,4 supporting the results we obtained with Reed and Vinson's method. In summary, the larger ascending targets did not produce RM, whereas the smaller ones did. This pattern is clearly consistent with impetus predictions.

An alternative interpretation of the above results is that participants know that a greater mass rises faster but are unable to evaluate the net effect of greater mass and greater air resistance on the movement of a larger object, assuming incorrectly that the effect of air resistance on a larger target would exceed the effect of increased mass. However, this interpretation of the results seems implausible for two reasons. First, if people incorrectly assume that the effect of larger size (and increased air resistance) is greater than the effect of increased mass, they should produce less RM for larger descending objects, as well as for larger ascending objects. However, Hubbard (1997) found that participants show more RM for larger descending objects than for smaller descend-

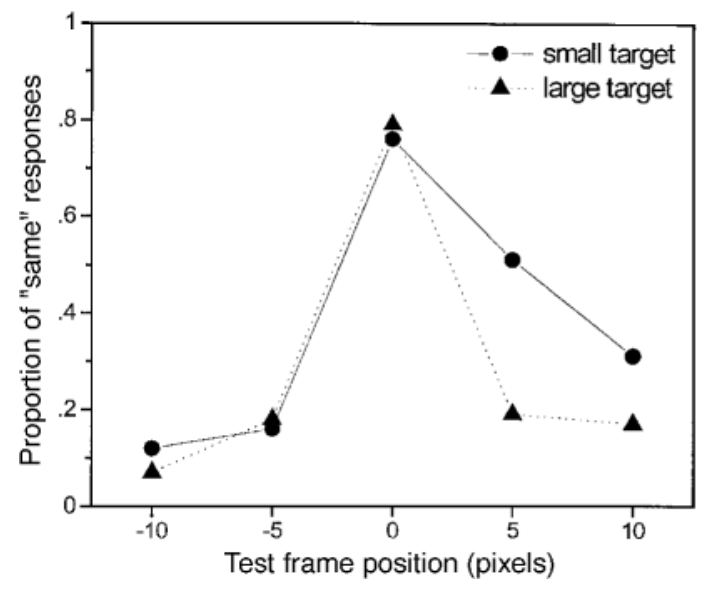

Figure 1. Mean proportion of same responses for the two targets at different shifts of the test frame in Experiment 1. 
ing objects. Only impetus theory predicts that, for descending motion, the larger target moves faster and, for ascending motion, the larger target moves slower. Second, Cooper and Munger (1993) found that sequences depicting motion of a triangle in the direction of its pointed end did not produce larger memory distortions than did sequences showing motion of a triangle in the direction of its base. This result suggests that people do not represent significant effects of friction between the target and the background medium.

\section{Experiment 2}

The goal of Experiment 2 was to examine the explicit knowledge of physics novices about objects' motion under the influence of gravity. We predicted that students' naive beliefs about the effect of mass on ascension rate would be consistent with impetus ideas.

\section{Method}

Participants. The participants were 103 undergraduate psychology students recruited from the psychology subject pool at the University of California, Santa Barbara, who had not received physics instruction at the college level. Ninety-three participants completed a written questionnaire regarding their beliefs about ascending and descending objects. Ten additional students were interviewed regarding these beliefs.

Questionnaire. The questionnaire consisted of five questions assessing participants' conceptual knowledge about motion (see the Appendix). The participants were asked to indicate which object (heavier or lighter) would fall or rise faster in the presence or absence of air resistance. They were also asked to draw and name all the forces, if any, acting upon a rising object.

Procedure. Ninety-three participants were tested in groups of about 30 . On average, it took 5-10 min to complete the questionnaire, but the participants were not placed under any time restriction. Ten additional participants were interviewed on the same questions presented in the written questionnaire. During the interviews, they were asked to think aloud while answering the questions.

\section{Results and Discussion}

The percentage of participants choosing each response option to the first four questions are shown in
Table 2. A large proportion of students indicated correctly that, in the absence of friction, all physical objects, regardless of mass, fall with the same acceleration. However, more than half of the participants indicated that, for ascending motion, even in the absence of friction, a more massive object ascends more slowly. It is plausible that many participants have heard about Galileo's famous thought experiment that, in a vacuum, all objects fall with the same acceleration. It seems, however, that their knowledge does not transfer to the question regarding the acceleration of rising objects in a vacuum, which is just a mirror image of the problem with falling objects. The results for Question 3 are striking. Only 1 participant correctly indicated that, in the presence of friction, a less massive object ascends more slowly.

Chi-square analyses showed significant differences between students' responses on the questions related to downward and upward motion without air resistance $\left[\chi^{2}(1)=31.00, p<.001\right]$ and between the questions on downward and upward motion in the presence of air resistance $\left[\chi^{2}(1)=44.00, p<.001\right]$. These results indicate that students' explicit beliefs are close to impetus theory, which predicts the same outcome for the effect of mass on ascension rate regardless of presence or absence of air resistance (see Table 1).

On Questions 2, 3, and 4, impetus and Newtonian theories make different predictions. In these questions, we used the chi-square goodness-of-fit test to examine whether the participants were more likely to choose the answer consistent with impetus than the answer consistent with Newtonian theory. This was the case $\chi^{2}(1)=$ $25.82, p<.001$, for Question 2; $\left[\chi^{2}(1)=84.05, p<.0001\right.$ for Question 3; and $\chi^{2}(1)=4.74, p<.03$, for Question 4].

In Question 5, the students were asked to draw the forces acting on a rising object. Ninety-two students indicated that two forces act on a rising object-the force of gravity that acts downward and an upward force with which the ball was thrown (reflecting impetus theories). Only 1 student indicated correctly that only one force,

Table 2

The Percentage of Participants Choosing Each Response to the First Four Questions of the Questionnaire in Experiment 2

\begin{tabular}{lccc}
\hline & \multicolumn{3}{c}{ Response } \\
\cline { 2 - 4 } \multicolumn{1}{c}{$\begin{array}{c}\text { Question } \\
\text { The Lighter Ball Will } \\
\text { Reach the Ground } \\
\text { (the Height) First }\end{array}$} & $\begin{array}{c}\text { The Balls Will Reach } \\
\text { the Ground (the Height) } \\
\text { at the Same Time }\end{array}$ & $\begin{array}{c}\text { The Heavier Ball Will } \\
\text { Reach the Ground (the } \\
\text { Height) First }\end{array}$ \\
\hline $\begin{array}{l}\text { Q1 (downward } \\
\text { motion with air } \\
\text { resistance) }\end{array}$ & 0 & 35.5 & 64.5 (N\&I) \\
$\begin{array}{l}\text { Q2 (downward } \\
\text { motion, no air } \\
\text { resistance) }\end{array}$ & 0 & $76.3(\mathrm{~N})$ & 23.7 (I) \\
$\begin{array}{l}\text { Q3 (upward motion } \\
\text { with air resistance) }\end{array}$ & $93.5(\mathrm{I})$ & & $1.1(\mathrm{~N})$ \\
$\begin{array}{l}\text { Q4 (upward } \\
\text { motion, no air } \\
\text { resistance) }\end{array}$ & $61.3(\mathrm{I})$ & $38.7(\mathrm{~N})$ & 0 \\
\hline
\end{tabular}

Note- $\mathrm{N}$ and I indicate responses in accord with Newtonian or impetus theories, respectively. N\&I indicates responses that are consistent with predictions of both Newtonian and impetus theories. 
the force of gravity, acts on a rising object. These results are consistent with previous studies (e.g., Clement, 1982; Viennot, 1979) and indicate the wide acceptance of impetus beliefs regarding the motion of objects in a gravitational field.

Informal interviews with 10 participants showed that they were susceptible to impetus beliefs. When asked to predict which of two ascending objects (a heavier or a lighter object) reaches a certain height first, all 10 participants stated that the lighter one does and explicitly stated that the heavier object ascends more slowly because it has to overcome a larger force of gravity. When asked what forces act on a rising object, all 10 participants stated that both gravity and the force of the throw act on the object. According to these participants, the force of the throw overcomes gravity, and when it is exhausted, the object begins to fall. The participants referred to their everyday experience that a more massive object should be thrown with a greater force than a smaller one in order to overcome the force of gravity. On the basis of the above experience, they made the erroneous conclusion that a more massive object ascends more slowly. Students' explanations were therefore consistent with fundamental ideas of impetus theory that (1) an internal force (the force of the throw) imparted to an object keeps it in rising motion and (2) this internal force is counteracted by gravity, which is greater for the heavier object and thus moves the heavier object downward faster.

The finding that almost all the students participating in Experiment 2 were susceptible to impetus beliefs suggests that, in the absence of specific contextual experience and physics training, people consistently fall back on impetus notions. In everyday life, a situation in which two different masses begin to rise with the same initial velocity is rare and less familiar than a situation in which two different masses begin to fall with the same initial velocity (equal to zero). The persistence and tenacity of naive beliefs exhibited by students seems to come from

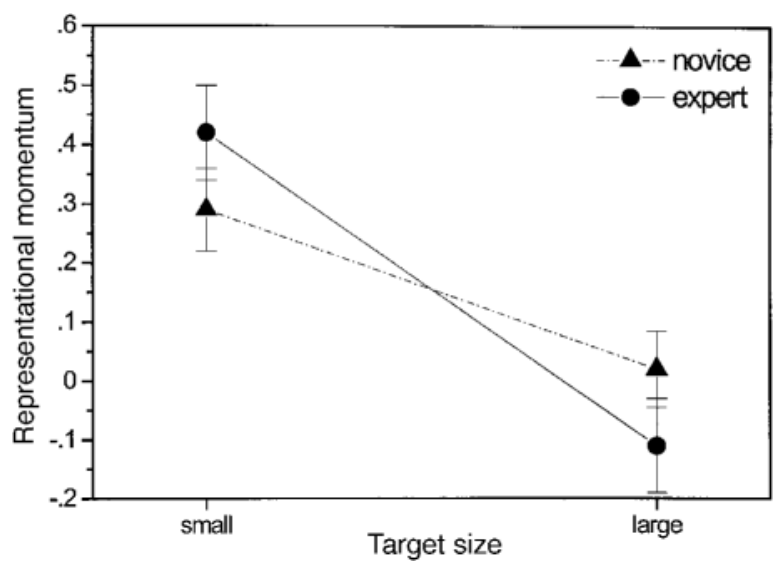

Figure 2. The size of the representational momentum effect for small and large targets for experts and novices in Experiment 3. Error bars represent standard errors. properties of our perceptual-representational system, which relies on impetus as a default heuristics.

\section{Experiment 3}

The goal of Experiment 3 was to examine how explicit knowledge of physics laws affects RM for ascending objects.

\section{Method}

Participan ts. There were 23 participants in the experiment. Eighteen participants were undergraduate psychology students recruited from the psychology subject pool at the University of California, Santa Barbara. Nine were true novices- that is, they had not taken any physics courses at either high school or college level; the other 9 students had taken at least one course in physics (mechanics) at the college level. In addition, 5 physics experts (graduate students and post-doctoral researchers with at least an undergraduate degree in physics) participated in the experiment.

The participants were pretested on the questionnaire used in Experiment 2 , which included only those questions asking which of two objects with different density but the same size would rise or fall faster. They were not pretested on the questions asking about objects of the same density, but different sizes, so that the questions would not affect their RM responses. All the participants were naive to the research hypothesis until after their data had been collected. All the participants chosen for the novice group (those without physics training) stated, in this pretest, that the lighter ascending object would rise more quickly — that is, their predictions were consistent with impetus theory. The undergraduate students with a physics background and the physics experts chosen for the purpose of this experiment were those who correctly stated, in the pretest, that the more massive object would rise more quickly, since it was less influenced by the force of friction. In addition, all the participants were interviewed after the RM trials and were asked explicitly which of two objects of the same density, but different sizes, would rise or fall faster. The novices all answered these questions according to impetus beliefs, and the students with a physics background and the physics experts all answered them correctly.

Stimuli and Apparatus. The stimuli and apparatus used in Experiment 3 were similar to those used in Experiment 1. On half the trials the target was large, and on half the trials the target was small. As in Experiment 1, the ascending target crossed approximately half a screen (5 in.) before it vanished. In contrast to Experiment 1 , we presented only the 5-pixel shift in Experiment 3, since this was the amount of displacement that showed the greatest memory distortions in Experiment 1. That is, the square depicted in the test frame was in the same position as the final square, was shifted 5 pixels backward in the direction of motion, or was shifted 5 pixels forward in the direction of motion.

Procedure. The procedure used in Experiment 3 was similar to that used in Experiment 1. The participants were first given 12 practice trials at the beginning of the session, drawn randomly from the experimental trials. They were given the same instructions as those in Experiment 1. Each participant received 20 blocks of 12 trials.

\section{Results and Discussion}

The mean proportion of same responses for different shifts of the test frame are shown in Table 3. The RM effect was calculated for each participant (using Reed \& Vinson's, 1996, formula). Since no significant differences were found between the RM effects shown by physics experts and undergraduate students with physics knowledge $[F(1,12)=0.27, p=.61$, for the large target, and $F(1,12)=0.04, p=.85$, for the small target], they 
Table 3

Mean Proportion of the Same Responses in Experiment 3 for Each Group of Participants for Targets of Different Sizes

\begin{tabular}{|c|c|c|c|c|c|c|}
\hline \multirow[b]{3}{*}{ Group and Target Size } & \multicolumn{6}{|c|}{ Mean Proportion of the Same Responses } \\
\hline & \multicolumn{2}{|c|}{-5-Pixel Shift } & \multicolumn{2}{|c|}{0.00} & \multicolumn{2}{|c|}{+5 -Pixel Shift } \\
\hline & $M$ & $S D$ & $M$ & $S D$ & $M$ & $S D$ \\
\hline \multicolumn{7}{|l|}{ Small } \\
\hline Group I (experts) & 0.12 & 0.2 & 0.78 & 0.2 & 0.54 & 0.2 \\
\hline Group II (novices) & 0.18 & 0.2 & 0.73 & 0.2 & 0.47 & 0.2 \\
\hline \multicolumn{7}{|l|}{ Large } \\
\hline Group 1(experts) & 0.28 & 0.1 & 0.75 & 0.1 & 0.17 & 0.1 \\
\hline Group II (novices) & 0.20 & 0.2 & 0.82 & 0.2 & 0.22 & 0.2 \\
\hline
\end{tabular}

Note-The " -5 pixel shift" refers to the shift of the test frame in the opposite direction of implied motion (downward shift). The " +5 -pixel shift" refers to the shift of the test frame in the direction of the implied motion (upward shift).

were pooled into one expert group. Also, no significant difference was found between this expert group and the novice group: $[F(1,21)=0.04, p=.85]$. The participants with correct physics knowledge produced similar effects of target size on RM to those for the participants with incorrect physics knowledge. The large ascending target produced significantly less RM than did the smaller ascending target $[F(1,21)=14.01, p<.001]$. The interaction between the target size and group was insignificant $[F(1,21)=0.27, p=.60]$. Figure 2 illustrates the RM effects by group and target size.

The results of this experiment support the idea that explicit and implicit knowledge are dissociated and that our implicit knowledge remains impenetrable by our abstract conceptual knowledge. Regardless of physics expertise or accuracy of explicit knowledge, people show a larger RM effect for smaller targets than for larger targets. It seems that both experts and novices possess the same set of impetus ideas on a perceptual level, although experts can successfully suppress these false beliefs at a conceptual level.

\section{Discussion of Empirical Results}

In summary, Experiments 1 and 3 provided evidence that when people are presented with an apparent motion display showing an ascending object, the size (implied mass) of the object influences the amount of RM observed. More RM is shown for small objects than for large objects. That is, RM effects conform to impetus predictions. Experiment 2 showed that novices' explicit judgments of the movement of ascending objects also conform to impetus principles. Finally, Experiment 3 showed that although people with physics training make correct explicit judgments about the effects of mass on ascending objects, their implicit knowledge, as revealed by RM, is not different from novices' knowledge and is consistent with impetus principles.

A previous study of the dissociation between explicit and implicit knowledge (Freyd \& Jones, 1994) for McCloskey's (1983) spiral tube problem parallels the present study. Both Freyd and Jones' study and our study doc- ument situations in which people's explicit knowledge is more correct than their implicit knowledge. In Freyd and Jones' study, greater RM was observed for the impetus (spiral) than for the Newtonian (straight) path. In our study, the greater RM in both novice and expert groups occurred for the lighter, rather than the heavier, object along the same path of motion (consistent with impetus and inconsistent with Newtonian principles). Simple pattern completion or good continuation of motion, as well as the idea that momentum occurs along a path that is consistent with the inducing display, cannot explain the RM effect in our study.

It might be argued that the incorrect implicit knowledge observed in Experiments 1 and 3 is due to the lack of realism of the display. That is, a more realistic display might evoke additional contextual cues and thus facilitate correct predictions. There are two ways in which the display could be considered to be unrealistic. First, this study, like Freyd's studies (e.g., Freyd, 1987) used an implied motion paradigm in which participants are shown a series of static frames, rather than a continuous animation. However, Hubbard's studies (e.g., Hubbard, 1990, 1997) show the same errors in RM for ascending targets, although the participants in those studies were presented with continuous animations. Second, RM studies often present participants with objects moving vertically with constant velocity, whereas, in fact, this is unrealistic for motion in a gravitational field. Nevertheless, our results show that incorrect RM effects occur even when the deceleration of ascending objects is modeled in the display. It is still possible, however, that correct implicit knowledge would be elicited in a much more realistic display, such as a videotape or a computer animation that shows continuous decelerating motion and much more contextual information.

The errors in implicit knowledge shown in RM studies seem to contradict results by Kaiser et al. (1985) that people can recognize the correct trajectory of objects coming from a curved tube when shown an animated display, even though they are subject to impetus misconceptions when shown a static display. The task in Kaiser 
et al.'s (1985) experiments, however, was very different from the RM task, in that it involved choosing between several possible trajectories and, most importantly, did not require immediate responses, as in the RM paradigm.

The result that explicit knowledge of physics does not influence implicit knowledge might be seen as contradictory to the results of Reed and Vinson (1996) showing that conceptual knowledge can influence RM. For example, Reed and Vinson showed that there is more upward RM for a picture of an ascending rocket than for a picture of an ascending steeple. However, Reed and Vinson's research relates to the influence of everyday knowledge on RM, whereas our research relates to knowledge of abstract physics laws. People have experience seeing movies of ascending rockets, and this type of contextual experience might be the source of their implicit knowledge in this case. That is, specific everyday experiences might lead to specific implicit knowledge, so that Reed and Vinson's results might reflect specific implicit knowledge about a rocket's motion, rather than effects of explicit conceptual knowledge. This hypothesis could be tested in a future experiment, which contrasts the effect of specific perceptual experience on the magnitude and direction of RM with the effect of physics instructions. For example, one could give one group of people experience catching balls emerging from a curved tube and another group physics instruction relative to this task and examine which of the treatments has a greater effect on RM.

\section{GENERAL DISCUSSION}

This paper makes three contributions to understanding explicit and implicit beliefs about motion and their relation to historical theories of motion. First, a review of the naive physics literature reveals that although people make accurate predictions about familiar situations, they appear to fall back on impetus beliefs in unfamiliar situations. Second, a review of the RM literature reveals that when impetus beliefs and Newtonian theory make different predictions about the movement of an object, RM is consistent with impetus beliefs. Third, new experimental results support the view that RM is consistent with impetus beliefs and show that explicit knowledge of the laws of physics does not influence RM.

There have been a number of previous studies suggesting a dissociation between explicit and implicit knowledge of the principles of physics. For instance, Hubbard suggests that implicit knowledge reflects internalization of invariant physical principles, whereas explicit knowledge about motion may be less accurate. Similarly, Krist et al. (1993) suggested that perceptually based knowledge is more accurate than verbal concepts of motion.

In contrast, our study has documented a situation in which the explicit knowledge of people with physics training is correct, but the implicit knowledge of these people is in accordance with impetus theories (see also Freyd \& Jones, 1994). In what situations might we expect implicit knowledge to be inaccurate? Our data and other studies (e.g., Catrambone et al., 1995; Kaiser et al., 1985) suggest that implicit knowledge might be inaccurate in situations that do not occur very often in everyday life and for which impetus theory and Newtonian physics make different predictions. People rarely see two different masses thrown up with the same initial velocity or balls being shot through curved tubes. It is plausible that if people have specific experience, such as watching a juggler juggle objects of different masses or an athlete using a slingshot, they might develop correct implicit knowledge for these situations (which would be reflected in correct RM effects).

Our study suggests that in unfamiliar situations, in which an immediate response is required, people apply impetus principles as a default heuristic. To extrapolate objects' motion on the basis of physical principles, one should have assessed and evaluated the presence and magnitude of such imperceptible forces as friction and air resistance operating in the real world. This would require a time-consuming analysis that is not always possible. In order to have a survival advantage, the process of extrapolation should be fast and effortless, without much conscious deliberation. Impetus theory allows us to extrapolate objects' motion quickly and without large demands on attentional resources. Reliance on the notion that objects somehow lose their internal energy as a result of motion allows us to respond quickly to a variety of situations in a world in which air, land, and water all offer air resistance or friction. Impetus seems to reflect the phenomenological aspects of our interaction with objects, and it is not surprising, then, that impetus beliefs are retained, even though they occasionally lead to errors in predictions. However, when people have physics knowledge and have enough time for analysis of the situation, they can apply correct physics principles to make judgments about moving objects and can avoid impetus predictions.

A central result of this research is that the reliance on impetus heuristics is not restricted to novices. Physics experts are also subject to the same biases when they think intuitively. These results are strikingly similar to Tversky and Kahneman's (1986) findings about the lack of difference between expert statisticians and novices on the conjunction fallacy. Tversky and Kahneman showed that people rely on a limited number of heuristic principles, which reduce the complex tasks of assessing probabilities. Statistically sophisticated researchers were found to be prone to the same biases and errors as novices, and their intuitive judgments were subject to similar fallacies in more intricate and less transparent problems. Similarly, McKenzie (1994) found that most people's intuitive strategies for covariation assessment and Bayesian inference are surprisingly efficient under many conditions, even though they can lead to serious errors in other conditions. McKenzie argues that since these intuitive strategies are much simpler than normative strategies, they may be the most efficient means of ensuring accurate judgments. In a similar way, impetus principles are 
much simpler than Newtonian principles and may be the most efficient means of ensuring fast and accurate predictions about objects' motion in most everyday situations.

This research can help explain the striking similarity between the historical development of the theory of motion and students' misconceptions about motion and the persistence of these misconceptions despite physics instructions. We propose that implicit knowledge might be the source of explicit knowledge in both historical development and the development of an individual. Thus, naive impetus explanations, as well as medieval impetus theory, might appear as an attempt to verbalize everyday perceptual experience. We also argue that specific perceptual experience might lead to correct implicit and explicit knowledge-for example, the knowledge that water emerging from a curved hose follows a straight trajectory (Kaiser et al., 1986). However, this knowledge will be very specific to the situation, and impetus ideas will still be used in very similar situations for which we do not have perceptual experience. This can lead to the mixture of correct and impetus ideas documented by diSessa (1983, 1988, 1993) and Ranney (1988, 1994).

In summary, we argue that impetus ideas reflect a systematic set of beliefs that people fall back on when they do not have specific contextual experience. Rather than characterizing knowledge as an idiosyncratic collection of ideas, we propose that both physics experts and novices possess the same set of implicit beliefs about motion. These implicit beliefs are based on perceptual experience and seem to exploit the "illusory" pattern that moving objects stop of their own accord as a result of losing their internal energy (impetus). We propose that these implicit beliefs can be suppressed in favor of correct physics principles, if one has learned these principles and has enough time to reflect, or as a result of specific contextual experience.

\section{REFERENCES}

BRonson, R. (1994). Schaum's outline of theory and problems of differential equations. New York: McGraw-Hill.

Catrambone, R., Jones, C. M., Jonides, J., \& Seifert, C. (1995). Reasoning about curvilinear motion: Using principles or analogy. Memory \& Cognition, 23, 368-373.

Clement, J. (1982). Students' preconceptions in introductory mechanics. American Journal of Physics, 50, 66-71.

Clement, J. (1983). A conceptual model discussed by Galileo and used intuitively by physics students. In D. Gentner \& A. Stevens (Eds.), Mental models (pp. 325-339). Hillsdale, New Jersey: Erlbaum.

Cooke, N. J., \& BREedin, S. D. (1994). Constructing naive theories of motion on the fly. Memory \& Cognition, 22, 474-493.

Cooper, L. A., \& Munger, M. P. (1993). Extrapolating and remembering positions along cognitive trajectories: Use and limitations of analogies to physical motion. In N. Eilan, R. McCarthy, \& B. Drewer (Eds.). Spatial representation: Problems in philosophy and psychology (pp. 112-131). Oxford: Blackwell.

DiJKSTERHUIS, E. J. (1961). The mechanization of the world picture. New York: Oxford University Press.

DISESSA, A. A. (1983). Phenomenology and the evolution of intuition. In D. Gentner \& A. Stevens (Eds), Mental models (pp. 5-33). Hillsdale, NJ: Erlbaum.

DISEssa, A. A. (1988). Knowledge in pieces. In G. Forman \& P. Pufall (Eds.), Constructivism in the computer age (pp.49-70). Hillsdale, NJ: Erlbaum.
DISEssa, A. (1993). Towards an epistemology of physics. Cognition \& Instruction. 10, 105-255.

Eckstein, S. G., \& Kozhevnikov, M. (1997). Parallelism in the development of children's ideas and the historical development of projectile motion theories. International Journal of Science Education, 19, 1057-1073.

EcKstein, S. G., \& Shemesh, M. (1993). Stage theory of the development of alternative conceptions. Journal of Research in Science Teaching, 30, 45-64.

Finke, R. A., \& FREYd, J. J. (1985). Transformation of visual memory induced by implied motions in pattern elements. Journal of Experimental Psychology: Learning, Memory, \& Cognition, 11, 780-794.

Finke, R. A., \& Freyd, J. J. (1989). Mental extrapolation and cognitive penetrability: Reply to Ranney and proposals for evaluative criteria. Journal of Experimental Psychology: General, 118, 403-408.

Finke, R. A., Freyd, J. J., \& Shyi, G. C.W. (1986). Implied velocity and acceleration induce transformations of visual memory. Journal of Experimental Psychology: General, 115, 175-188.

Fishbein, E., Stavy, R., \& Ma-Naim, H. (1989). The psychological structure of naive impetus conceptions. International Journal of Science Education, 11, 71-81.

Franklin, A. (1978). Inertia in the middle ages. Physics Teacher, 16, 201-208.

FreYd, J. J. (1987). Dynamic mental representations. Psychological Review, 94, 427-438.

FreYD, J. J. (1992). Dynamic representations guiding adaptive behavior. In D. Meyer \& S. Kornblum (Eds.), Time, action, and cognition: Towards bridging the gap (pp. 309-323). Dordrecht: Kluwer.

FrEYD, J. J. (1993). Five hunches about perceptual processes and dynamic representations. In D. Meyer \& S. Kornblum (Eds.), Attention and performance XIV: Synergies in experimental psychology, artificial intelligence, and cognitive neuroscience (pp. 99-119). Cambridge, MA: MIT Press.

Freyd, J. J., \& FinKe, R. A. (1984). Representational momentum. Journal of Experimental Psychology: Learning, Memory, \& Cognition, 10, 126-132.

Freyd, J. J., \& Johnson, J. Q. (1987). Probing the time course of representational momentum. Journal of Experimental Psychology: Learning, Memory, \& Cognition, 13, 259-268.

Freyd, J. J., \& JonES, K. T. (1994). Representational momentum for a spiral path. Journal of Experimental Psychology: Learning, Memory, \& Cognition, 20, 968-976.

Halloun, I. A., \& Hestenes, D. (1985). The initial knowledge state of college physics students. American Journal of Physics, 53, 1043-1055.

HubBARD, T. L. (1990). Cognitive representation of linear motion: Possible direction and gravity effects in judged displacement. Memory \& Cognition, 18, 299-309.

HubBard, T. L. (1995a). Cognitive representations of motion: Evidence for representational friction and gravity analogues. Journal of Experimental Psychology: Learning, Memory, \& Cognition, 21, 241254.

Hubbard, T. L. (1995b) Environmental invariants in the representation of motion: Implied dynamics and representational momentum, gravity, friction, and centripetal force. Psychonomic Bulletin \& Review, 2, 322-338.

HubBaRD, T. L. (1996). Representational momentum, centripetal force, and curvilinear impetus. Journal of Experimental Psychology: Learning, Memory, \& Cognition, 22, 1049-1060.

Hubbard, T. L. (1997). Target size and displacement along the axis of implied gravitational attraction: Effects of implied weight and evidence of representational gravity. Journal of Experimental Psychology: Learning, Memory, \& Cognition, 23, 1484-1493.

HubBARd, T. L. (1998). Representational momentum and other displacement in memory as evidence for nonconscious knowledge of physical principles. In S. Hameroff, A Kaszniak, \& A. Scott (Eds.), Toward a science of consciousness: II. The 1996 Tucson discussion and debates (pp. 505-512). Cambridge, MA: MIT Press.

Hubbard, T. L., \& Bharucha, J. J. (1988). Judged displacement in apparent vertical and horizontal motion. Perception \& Psychophysics, 44, 211-221.

Jones, L. A. (1986). Perception of force and weight: Theory and research. Psychological Bulletin \& Review, 100, 29-42. 
Kaiser, M. K., Jonides, J. \& Alexander, J. (1986). Intuitive reasoning about abstract and familiar physics problems. Memory \& Cognition, 14, 308-312.

Kaiser, M. K., Proffitt, D. R., \& Anderson, K. (1985). Judgments of natural and anomalous trajectories in the presence and absence of motion. Journal of Experimental Psychology: Learning, Memory, \& Cognition, 11, 795-803.

Kaiser, M. K., Proffitt, D. R. Whelan, S. M., \& Hecht, H. (1992). Influence of animation on dynamical judgments. Journal of Experimental Psychology: Human Perception \& Performance, 18, 669689.

Koseleff, P. (1957). Studies in the perception of heaviness. Acta Psychologica, 13, 242-252.

Krist, H., Fieberg, E. L., \& Wilkening, F. (1993). Intuitive physics in action and judgment: The development of knowledge about projectile motion. Journal of Experimental Psychology: Learning, Memory, \& Cognition, 19, 952-966.

Masin, S. C., \& Crestoni, L. (1988). Experimental demonstration of the sensory basis of the size-weight illusion. Perception \& Psychophysics, 44, 309-312.

McCloskey, M. (1983). Naive theories of motion. In D. Gentner \& A. Stevens (Eds.), Mental models (pp. 229-324), Hillsdale, NJ:Erlbaum.

McCloskey, M., Caramazza, A., \& Green, B. (1980). Curvilinear motion in the absence of external forces: Naive beliefs about the motion of objects. Science, 210, 1139-1141.

McCloskey, M., \& Kohl, D. (1983). Naive physics: The curvilinear impetus principles and its role in interactions with moving objects. Journal of Experimental Psychology: Learning, Memory, \& Cognition, 9, 146-156.

McCloskey, M., Washburn, A., \& Felch, L. (1983). Intuitive physics: The straight-down belief and its origin. Journal of Experimental Psychology: Learning, Memory, \& Cognition, 9, 636-649.

McKenzie, C. R. M. (1994). The accuracy of intuitive judgment strategies: Covariation assessment and Bayesian inference. Cognitive Psychology, 26, 209-239.

OGborn, J. (1985). Understanding students' understandings: An example from dynamics. European Journal of Science Education, 7, 141-150.

RANney, M. (1988, November). Contradictions and reorganizations among naive conceptions of ballistics. Paper presented at the 29th Annual Meeting of the Psychonomic Society, Chicago.

RANNEY, M. (1994). Relative consistency and subjects" "theories" in domains such as naive physics: Common research difficulties illustrated by Cooke and Breedin. Memory \& Cognition, 22, 494-502.

Ranney, M., \& Thagard, P. (1988). Explanatory coherence and belief revision in naive physics. In Proceedings of the Tenth Conference of the Cognitive Science Society (pp. 426-432), Hillsdale, NJ: Erlbaum.

ReEd, C. L., \& VInson, N. G. (1996). Conceptual effects on represen- tational momentum. Journal of Experimental Psychology: Human Perception \& Performance, 22, 839-850.

Tversky, A., \& Kahneman, D. (1986). Judgment under uncertainty: Heuristics and biases. In H. R. Arkes \& K. R. Hammond (Eds.), Judgment and decision making: An interdisciplinary reader (pp. 3855). Cambridge: Cambridge University Press.

Verfaillie, K., \& D'Ydewalle, G. (1991). Representational momentum and event course anticipation in the perception of implied periodical motions. Journal of Experimental Psychology: Learning, Memory, \& Cognition, 17, 302-313.

Viennot, L. (1979). Spontaneous reasoning in elementary dynamic. European Journal of Science Education, 1, 205-221.

\section{NOTES}

1. Impetus notions are not specified at such a level of detail that they make a single prediction in a given situation. As a result, different predictions about a given situation can all reflect impetus ideas. For instance, for a horizontally launched projectile, impetus theory is consistent with a number of different predicted trajectories: (1) a square-like trajectory where the object first moves in a straight line and then (when its impetus completely dissipates) begins to fall straight down, (2) a trajectory where the object moves in a straight line and then (when its impetus falls below some critical level) begins to fall in a parabolic arc, or (3) a correct parabolic trajectory (when its impetus dissipates gradually). In contrast, Newtonian predictions are consistent only with one possible trajectory for projectiles, which is a parabolic arc.

2 . The velocity-damping coefficient in the motion equation (see Equation 1) reflects the effect of air resistance on an object's motion, and it is equal to $k=k * / m$, where $k^{*}$ is the air resistance coefficient and $m$ is the mass of the object. The more massive an object, the smaller the velocity-damping coefficient will be (and, consequently, the smaller the effect of air resistance on the object's motion). Even in the case of two objects of the same density and different sizes, the effect of increased mass $\left(m \sim \rho \mathrm{L}^{3}\right.$, where $\rho$ is an object's density and $L$ is the length of its characteristic side) will always exceed the effect of increased air resistance coefficient $\left(k^{*} \sim L^{2}\right)$. Therefore, a more massive object will rise or fall at a higher rate than a less massive object owing either to its larger size (when the density is the same) or to larger density (when the size is the same).

3. The order of blocks by shift size was not counterbalanced. However, it is unlikely that this affects the results, since Freyd (1987) found that practice and feedback do not influence RM.

4. Because the greatest memory displacement was shown on the 5pixel shift of the test frame in Experiment 1, we focus on the 5-pixel shift of the test frame and use only Reed and Vinson's (1996) method in Experiment 3 .

\section{APPENDIX \\ Questionnaire Used in Experiment 2}

Two metal balls are the same size, but one weighs twice as much as the other. If they are dropped from the same height at the same time

1. Take air resistance into account:

(a) the heavier ball will reach the ground first;

(b) the lighter ball will reach the ground first;

(c) the two balls will reach the ground at the same time.

2. Ignore air resistance:

(a) the heavier ball will reach the ground first;

(b) the lighter ball will reach the ground first;

(c) the two balls will reach the ground at the same time.

Two metal balls are the same size, but one weighs twice as much as the other. Both are thrown straight up with the same initial velocity. The time it takes the balls to reach a certain height $H$ will be: 


\section{APPENDIX (Continued)}

3. Take air resistance into account:

(a) shorter for the heavier ball;

(b) shorter for the lighter ball;

(c) about the same time for both balls.

4. Ignore air resistance:
(a) shorter for the heavier ball;
(b) shorter for the lighter ball;
(c) about the same time for both balls.

5. A ball is thrown from point A straight up into the air and caught at point E. Draw one or more arrows showing the direction of each force acting on the ball when it is at point B. (Draw longer arrows for larger forces.)

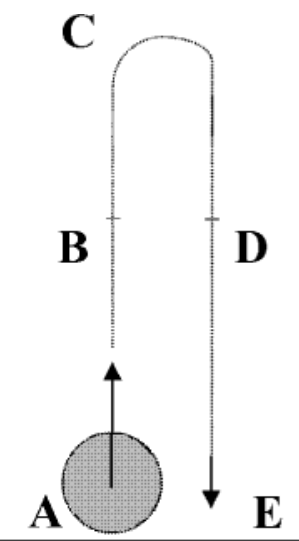

(Manuscript received July 14, 1999;

revision accepted for publication November 20, 2000.) 